\title{
A note on compactifications on Spin(7)-holonomy manifolds
}

\author{
Katrin Becker \\ California Institute of Technology 452-48, Pasadena, CA 91125 \\ CIT-USC Center for Theoretical Physics \\ E-mail: 'beckerkètheory.caltech.edù'
}

\begin{abstract}
In this note we consider compactifications of $\mathcal{M}$-theory on $\operatorname{Spin}(7)$-holonomy manifolds to three-dimensional Minkowski space. In these compactifications a warp factor is included. The conditions for unbroken $N=1$ supersymmetry give rise to determining equations for the 4-form field strength in terms of the warp factor and the self-dual 4-form of the internal manifold.
\end{abstract}

KEYwords: 
Warped compactifications of $\mathcal{M}$-theory and $\mathcal{F}$-theory have attracted recently much attention in connection to confining gauge theories [1] theoretic realization of the Randall-Sundrum scenario [ [6. the conditions for unbroken supersymmetry for compactifications on Calabi-Yau 4folds were found. These are manifolds that admit two covariantly constant spinors. It has recently been shown that the model considered by Klebanov and Strassler [3:]. can be obtained from the general type of solutions presented in 9.9.

From this perspective it is rather interesting to understand the physics of warped compactifications of $\mathcal{M}$-theory. ${ }^{1}$ In this note we would like to describe compactifications of $\mathcal{M}$-theory on Spin(7)-holonomy manifolds. These are eight-manifolds that admit only one covariantly constant Majorana-Weyl spinor which arises from the the decomposition $8_{c} \rightarrow 7 \oplus 1$. Therefore these compactifications will give rise to $N=1$ theories in three dimensions while the models considered in $[9]$ had an $N=2$ supersymmetry. These theories are interesting because they cannot be obtained from a compactification of any supersymmetric four-dimensional theory on an $S^{1}$. In fact, a theory with $N=1$ in $d=4$ would yield an $N=2$ theory in three-dimensions [i]

Spin(7)-holonomy manifolds can be treated in a similar way as the Calabi-Yau 4 -fold case considered in [6. conventions of

To derive the conditions following from unbroken supersymmetry we start with the supersymmetry transformation of the gravitino in eleven-dimensional supergravity

$$
\delta \Psi_{M}=\nabla_{M} \eta-\frac{1}{288}\left(\Gamma_{M}^{P Q R S}-8 \delta_{M}^{P} \Gamma^{Q R S}\right) F_{P Q R S} \eta=0 .
$$

We make the following ansatz for the metric

$$
g_{M N}(x, y)=\Delta^{-1}(y)\left(\begin{array}{cc}
g_{\mu \nu}(x) & 0 \\
0 & g_{m n}(y)
\end{array}\right)
$$

where $x$ are the coordinates of the external space labeled by the indices $\mu, \nu, \ldots$ and $y$ are the coordinates of the internal manifold labeled by $m, n, \ldots \Delta=\Delta(y)$ is the warp factor.

The eleven-dimensional spinor $\eta$ is decomposed as

$$
\eta=\epsilon \otimes \xi
$$

where $\epsilon$ is a three-dimensional anticommuting spinor and $\xi$ is an eight-dimensional Majorana-Weyl spinor. Furthermore we make the decomposition of the gamma matrices

$$
\begin{gathered}
\Gamma_{\mu}=\gamma_{\mu} \otimes \gamma_{9}, \\
\Gamma_{m}=1 \otimes \gamma_{m},
\end{gathered}
$$

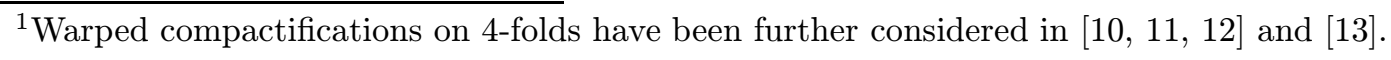


where $\gamma_{\mu}$ and $\gamma_{m}$ are the gamma matrices of the external and internal space respectively. We choose the matrices $\gamma_{m}$ to be real and antisymmetric. $\gamma_{9}$ is the eight-dimensional chirality operator which anti-commutes with all the $\gamma_{m}$ 's.

In compactifications with maximally symmetric three-dimensional space-time the non-vanishing components of the 4 -form field strength $F_{4}$ are

$$
\begin{aligned}
& F_{m n p q} \text { arbitrary } \\
& F_{\mu \nu \rho m}=\epsilon_{\mu \nu \rho} f_{m}
\end{aligned}
$$

where $F_{m n p q}$ and $f_{m}$ will be determined from the conditions following from unbroken supersymmetry and $\epsilon_{\mu \nu \rho}$ is the Levi-Civita tensor of the three-dimensional external space. The external component of the gravitino supersymmetry transformation is given by the following expression

$$
\begin{aligned}
\delta \psi_{\mu}= & \nabla_{\mu} \eta-\frac{1}{288} \Delta^{3 / 2}\left(\gamma_{\mu} \otimes \gamma^{m n p q}\right) F_{m n p q} \eta+ \\
& +\frac{1}{6} \Delta^{3 / 2}\left(\gamma_{\mu} \otimes \gamma^{m}\right) f_{m} \eta+\frac{1}{4} \partial_{n}(\log \Delta)\left(\gamma_{\mu} \otimes \gamma^{n}\right) \eta
\end{aligned}
$$

where we have used a positive chirality eigenstate $\gamma_{9} \xi=\xi$. Considering negative chirality spinors corresponds to an eight-manifold with a reversed orientation [i] $\left.{ }_{1}^{1} \bar{y}_{1}\right]$. Since we would like to compactify $\mathcal{M}$-theory to three-dimensional Minkowski space we impose the condition

$$
\nabla_{\mu} \epsilon=0
$$

The external component of supersymmetry is then reduced to the equation

$$
T \xi=0 \quad \text { with } T=F_{m n p q} \gamma^{m n p q}-48\left(f_{n}-\partial_{n} \Delta^{-3 / 2}\right) \gamma^{n} .
$$

Taking into account that $\xi$ is Weyl we conclude that the external component of $F_{4}$ can be expressed in terms of the warp factor

$$
F_{\mu \nu \rho m}=\epsilon_{\mu \nu \rho} \partial_{m} \Delta^{-3 / 2}
$$

while the internal component of $F_{4}$ (which we denote by $F$ ) is constrained to satisfy

$$
F_{m n p q} \gamma^{m n p q} \xi=0 \text {. }
$$

The analysis of the internal components of supersymmetry can be performed as in [9.9.]. We find that in terms of the transformed quantities

$$
\begin{aligned}
\tilde{g}_{m n} & =\Delta^{-3 / 2} g_{m n}, \\
\tilde{\xi} & =\Delta^{1 / 4} \xi,
\end{aligned}
$$

the internal component of the gravitino transformation is given by

$$
\tilde{\nabla}_{m} \tilde{\xi}+\frac{1}{24} \Delta^{-3 / 4} F_{m} \tilde{\xi}=0
$$


The metric $\tilde{g}_{m n}$ describes the Spin(7)-holonomy manifold. These manifolds are Ricci flat and they admit one covariantly constant spinor which satisfies

$$
\tilde{\nabla}_{m} \tilde{\xi}=0 .
$$

Therefore we see that $F$ has to satisfy

$$
F_{m n p q} \tilde{\gamma}^{n p q} \tilde{\xi}=0
$$

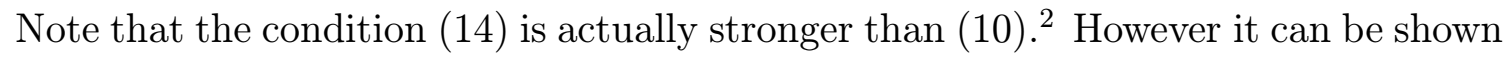

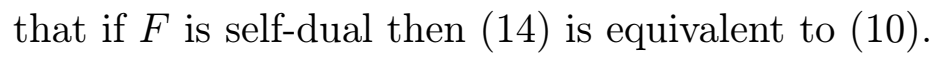

The proof that $F$ is self-dual goes as follows. From (1) we obtain the equation

$$
F_{m n p q} \tilde{\xi}^{T}\left\{\tilde{\gamma}^{n p q}, \tilde{\gamma}_{a b c}\right\} \tilde{\xi}=0 .
$$

To further evaluate (1) (1) $\left.\overline{1}_{1}^{1}\right)$ we note that we can construct covariantly constant $p$-forms in terms of the eight-dimensional spinor $\tilde{\xi}$

$$
\omega_{a_{1} \ldots a_{p}}=\tilde{\xi}^{T} \tilde{\gamma}_{a_{1} \ldots a_{p}} \tilde{\xi}
$$

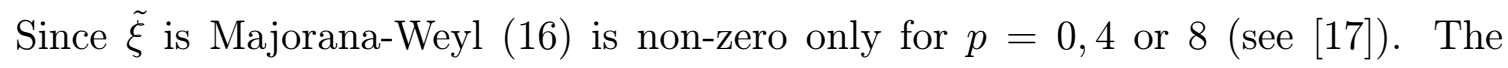
$\operatorname{Spin}(7)$ calibration is then given by the closed self-dual 4 -form

$$
\Phi_{m n p q}=\tilde{\xi}^{T} \tilde{\gamma}_{m n p q} \tilde{\xi}
$$

If we would have considered negative chirality spinors this form would be anti-self-

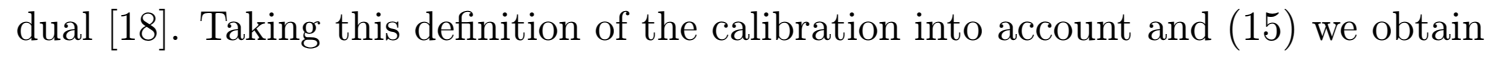

$$
F_{m n p q}=\frac{3}{2} F_{a b m[n} \Phi_{p q]}^{a b} .
$$

By considering the quantity

$$
F_{m n p q} \tilde{\xi}^{T}\left\{\tilde{\gamma}_{a b c d}, \tilde{\gamma}^{m n p q}\right\} \tilde{\xi}=0,
$$

we can derive the condition

$$
\star F_{m n p q}+F_{m n p q}=3 F_{a b[m n} \Phi_{p q]}^{a b},
$$

where by $\star$ we mean the Hodge dual with respect to the metric of the eight-dimensional internal space. Antisymmetrizing the right hand side of (1) $\left(\begin{array}{l}1 \\ 1\end{array}\right)$ over the indices $(m n p q)$ and comparing with $(\underline{2} \overline{2} \overline{0})$ we see that $F$ satisfies the self-duality condition

$$
F=\star F
$$

This self-duality condition can also be obtained from the equation of motion of $F$ by using the explicit form of the external component of $F_{4}[i \overline{1} \bar{i}]$.

\footnotetext{
${ }^{2}$ This equation has been noticed before in [1] $\left.\overline{6}_{i}\right]$.
} 
Taking this self-duality condition into account, we next would like to show that the constraint (1) coming from the internal component of supersymmetry is equivalent to the condition $\left(\overline{1}_{1}^{\overline{1}} \overline{0}_{1}\right)$. For this purpose a useful identity to consider is

$$
F_{m} F^{m}=-\frac{1}{8} \mathcal{F}^{2}-3 F_{m n p q}\left(F^{m n p q}-\star F^{m n p q}\right),
$$

where $\mathcal{F}=F_{m n p q} \tilde{\gamma}^{m n p q}$. Since $F$ is self-dual the second term on the right hand

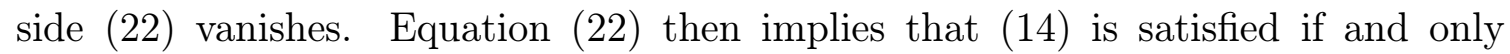
if $\left(\overline{1} \overline{0} \bar{O}_{1}^{\prime}\right)$ is fulfilled.

To see the conditions imposed by $\left(\mathbf{i} \overline{0}_{-1}\right)$ on our flux we note that Fierz rearrangements imply

$$
F_{m n p q} \tilde{\gamma}^{m n p q} \tilde{\xi}=F_{m n p q} \Phi^{m n p q} \tilde{\xi}-F_{m n p r} \Phi_{s}{ }^{m n p} \tilde{\gamma}^{r s} \tilde{\xi}
$$

The condition for unbroken supersymmetry states that the left hand side of (20.3i vanishes. After multiplying this equation by $\tilde{\xi}^{T}$ we get

$$
F \wedge \Phi=0
$$

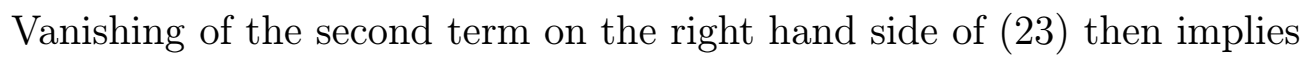

$$
\omega_{r s} \tilde{\gamma}^{r s} \tilde{\xi}=0
$$

where we have defined a 2 -form $\omega$ as

$$
\omega=\frac{1}{2} F_{m n p r} \Phi_{s}{ }^{m n p} d x^{r} \wedge d x^{s} .
$$

The spinors $\tilde{\gamma}^{r s} \tilde{\xi}$ are not independent [i] $\left.\overline{1} \overline{1}\right]$. To satisfy $\left(\underline{2}^{2} \overline{5_{1}^{\prime}}\right) \omega$ has to obey the selfduality constraint

$$
\frac{1}{2} \Phi^{r s p q} \omega_{p q}=\lambda \omega^{r s}
$$

with $\lambda=1$. But by taking the relation

$$
\Phi^{m n p t} \Phi_{q r s t}=6 \delta_{q r s}^{m n p}-9 \delta_{[q}^{[m} \Phi^{n p]}{ }_{r s]}
$$

and the definition of $\omega$ into account it is easy to see that $\omega$ satisfies $\left(\overline{2}_{2}^{\overline{2}} \bar{z}_{1}\right)$ with $\lambda=-3$. Therefore we conclude

$$
\omega=0 \text {. }
$$

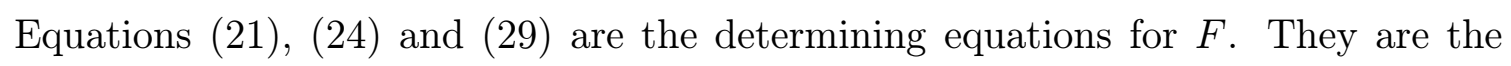
necessary and sufficient conditions for (1, 1 i $)$ to be satisfied.

After imposing the self-duality condition (1200i) takes the form

$$
F_{m n p q}=\frac{3}{2} F_{a b[m n} \Phi_{p q]}^{a b} .
$$

By contracting this equation with $\Phi^{n p q r}$ it is possible to show that (130ij) is equiv-

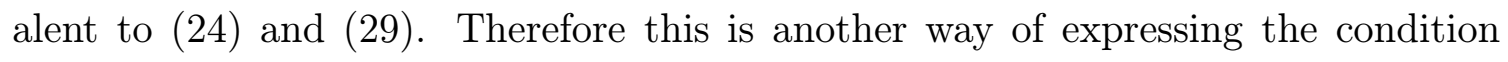


for unbroken supersymmetry. In this form the condition for unbroken supersymmetry is similar to the one satisfied by Yang-Mills fields $\mathcal{F}_{m n}$ for which the following equivalence relation holds [200]

$$
\mathcal{F}_{m n}=\frac{1}{2} \Phi_{m n p q} \mathcal{F}^{p q} \quad \Longleftrightarrow \quad \mathcal{F}_{m n} \tilde{\gamma}^{m n} \tilde{\xi}=0
$$

The determining equation for the warp factor is the fivebrane Bianchi identity which after using our solution for $F_{4}$ takes the same form as in [9.]]

$$
d \star d \log \Delta=\frac{1}{3} F \wedge F-\frac{2}{3}(2 \pi)^{4} X_{8} .
$$

For compactifications of $\mathcal{M}$-theory on $\operatorname{Spin}(7)$-holonomy manifolds we can expect to find non-vanishing expectation values for $F_{4}$ independently of the fact that the manifold is compact or not. This is different than the situation considered in [2] in which compactifications of $\mathcal{M}$-theory on seven-dimensional manifolds were considered and which only had non-vanishing expectation values for $F$ in the case that the internal manifold was non-compact.

For a compact $\operatorname{Spin}(7)$-holonomy manifold $K^{8}$ we can integrate $(\underline{3} \overline{2} \overline{2})$ and obtain the relation

$$
\int_{K^{8}} F \wedge F+\frac{1}{12} \chi=0
$$

where $\chi$ is the Euler number of the eight-manifold [9, 1220

To summarize we have found the following conditions for unbroken supersymmetry for compactifications of $\mathcal{M}$-theory on manifolds with $\operatorname{Spin}(7)$ holonomy to three-dimensional Minkowski space: the internal components of $F_{4}$ obey the con-

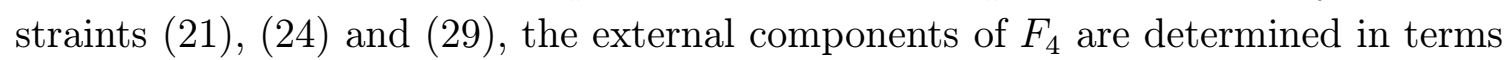
of the warp factor by (1, (19)

Concrete examples of compact Spin(7)-holonomy manifolds have been construc-

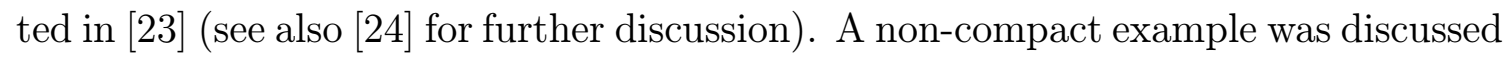
recently in $[2 \overline{2}]^{3}$ and was constructed in [1] in this case the explicit form of the metric is known and it takes the form of a quaternionic line bundle over a 4-sphere:

$$
d s_{8}^{2}=\alpha(r)^{2} d r^{2}+\beta(r)^{2}\left(\sigma^{i}-A^{i}\right)^{2}+\gamma(r)^{2} d \Omega_{4}^{2},
$$

where $\sigma_{i}$ are left-invariant 1-forms of $\mathrm{SU}(2), A^{i}$ are $\mathrm{SU}(2)$ Yang-Mills potentials on the unit 4-sphere whose metric is $d \Omega_{4}^{2}$. We will be following the notation of [2]-5i] and refer the reader to this work for further details.

In [25] an explicit computation of $F$ was done. In the following we would like to show that this solution satisfies our equations. This solution is anti-self-dual. This would correspond to choosing spinors with negative chirality (i.e. which satisfy $\left.\gamma_{9} \tilde{\xi}=-\tilde{\xi}\right)$ instead of the positive chirality spinors that we have used here. To show

\footnotetext{
${ }^{3}$ See also [i] $\left[\overline{1}_{i}\right]$.
} 
that equation ( $(2 \overline{4})$ is satisfied we need the explicit forms of $\Phi$ and $F$. They are given by

$$
\Phi=f_{1}^{\prime} d r \wedge \epsilon_{(3)}-\left(f_{1}+g_{1}\right) Y_{(4)}+g_{1}^{\prime} d r \wedge X_{3}-6 g_{1} \Omega_{(4)},
$$

with

$$
f_{1}=\frac{1}{5} c_{1}(1-6 z) z^{-6 / 5} \quad \text { and } \quad g_{1}=c_{1} z^{-6 / 5}
$$

while $F$ is given by

$$
F=f_{2}^{\prime} d r \wedge \epsilon_{(3)}-\left(f_{2}+g_{2}\right) Y_{(4)}+g_{2}^{\prime} d r \wedge X_{3}-6 g_{2} \Omega_{(4)},
$$

with

$$
f_{2}=\frac{1}{5}(z-6) z^{1 / 5} \quad \text { and } \quad g_{2}=z^{1 / 5} .
$$

Using this explicit forms for $F$ and $\Phi$ it is easy to see that $F \wedge \Phi$ is proportional to the quantity

$$
g_{1} f_{2}^{\prime}+g_{2} f_{1}^{\prime}+g_{1}^{\prime}\left(f_{2}+g_{2}\right)+g_{2}^{\prime}\left(f_{1}+g_{1}\right),
$$

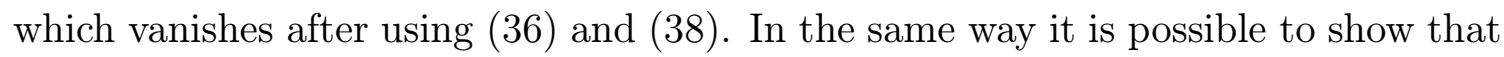
the 2-form $\omega$ vanishes. This is easily seen in the orthonormal basis $\hat{e}^{I}$ introduced in [i]̄i].

A superpotential in terms of the calibration describing compactifications on Spin(7)-holonomy manifolds has been conjectured in [1]2]. It would be interesting to see if the conditions for unbroken supersymmetry obtained in this paper can actually be derived from the superpotential presented in [1]2i].

\section{Acknowledgments}

I thank M. Becker, S. Gukov, J. Polchinski and E. Witten for useful discussions and correspondence. This work was supported by the U.S. Department of Energy under grant DE-FG03-92-ER40701.

\section{References}

[1] J. Polchinski and M.J. Strassler, The string dual of a confining four-dimensional gauge theory, hep-th/0003136!

[2] I.R. Klebanov and A.A. Tseytlin, Gravity duals of supersymmetric $\mathrm{SU}(N) \times \mathrm{SU}(N+M)$ gauge theories, №ul. Phys. B 578 - 2000$) 123$, [hep-th/0002159'.

[3] I.R. Klebanov and M.J. Strassler, Supergravity and a confining gauge theory: duality cascades and chisb-resolution of naked singularities, :

[4] M. Graña and J. Polchinski, Supersymmetric three-form flux perturbations on $A d S_{5}$,

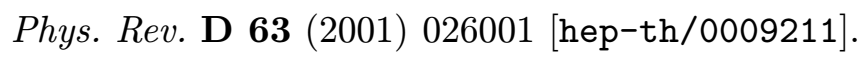


[5] S.S. Gubser, Supersymmetry and F-theory realization of the deformed conifold with three-form flux, hep-th/0010010.'.

[6] L. Randall and R. Sundrum, A large mass hierarchy from a small extra dimension, Phys. Rev. Lett. 83 (1999) 3370 [hep-th/9905221

[7] L. Randall and R. Sundrum, An alternative to compactification, : - .

[8] C.S. Chan, P.L. Paul and H. Verlinde, A note on warped string compactification, iNúcli: '.

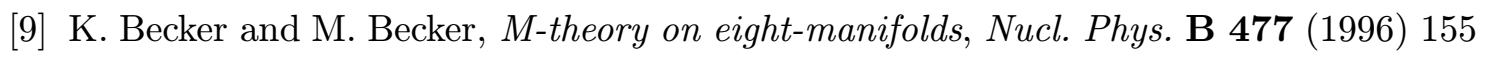
[hep-th/9605053in.

[10] S. Gukov, C. Vafa and E. Witten, CFT's from Calabi-Yau four-folds, 'Nucis. $584(2000) 69$.

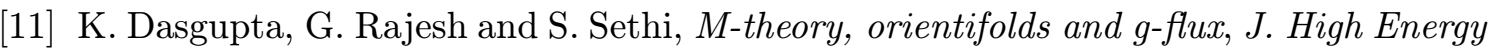
[ing-th/9908088'.

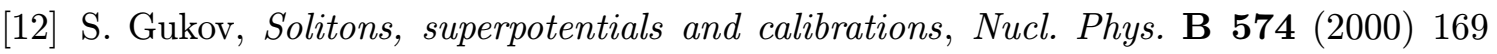
[hep-th/9911011.

[13] J.S. Gates, S. Gukov and E. Witten, Two two-dimensional supergravity theories from Calabi-Yau four-folds, Nucl. Phys. B 584 - 2000$)$ 109 thep-th/0005120il.

[14] C. Vafa, Evidence for F-theory, iNucl. Phys. B 469 11996$)$ 403; [hep-th/9602022].

[15] C.J. Isham and C.N. Pope, Nowhere vanishing spinors and topological obstruction to the equivalence of the NSR and GS superstrings, 'Class. and Quant. Grav. $\mathbf{5}(1 \overline{1} 88)$ : -

C.J. Isham, C.N. Pope and N.P. Warner, Nowhere-vanishing spinors and triality ro-

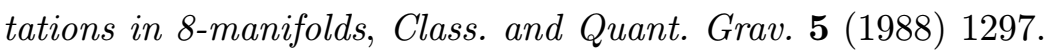

[16] S.W. Hawking and M.M. Taylor-Robinson, Bulk charges in eleven dimensions, ${ }^{\mathbf{P}}$ hys.'

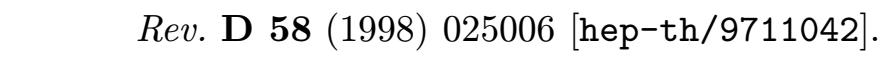

[17] G.W. Gibbons, D.N. Page and C.N. Pope, Einstein metrics on $S^{3}, R^{3}$ and $R^{4}$ bundles,

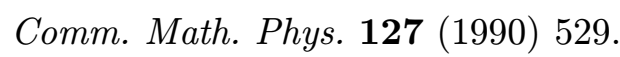

[18] J.A. Harvey and A. Strominger, Octonionic superstring solitons, 'L (1)

[19] M. Marino, R. Minasian, G. Moore and A. Strominger, Nonlinear instantons from

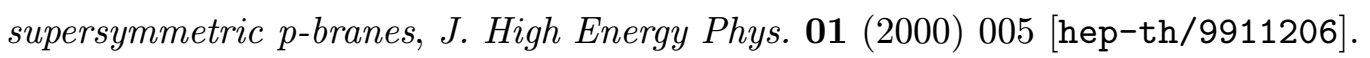

[20] E. Corrigan, C. Devchand, D.B. Fairlie and J. Nuyts, First order equations for gauge

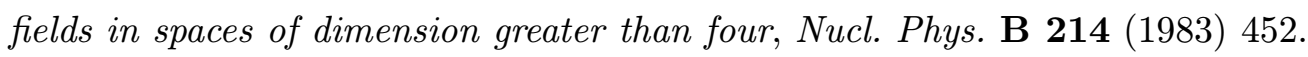


[21] K. Becker and M. Becker, Compactifying M-theory to four dimensions, ; - . Phys 11 12000$) 029$

[22] S. Sethi, C. Vafa and E. Witten, Constraints on low-dimensional string compactifications, Nucl. Phys. B 480 (1996) 213 [hep-th/9606122].

[23] D. Joyce, Compact 8-manifolds with holonomy Spin(7), Invent. Math. 123 (1996) 507.

[24] B.S. Acharya, On mirror symmetry for manifolds of exceptional holonomy, 'Nㅡㅁㅡ. 'P

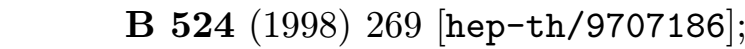

B.S. Acharya, J.M. Figueroa-O'Farril, C.M. Hull and B. Spence, Branes at conical singularities and holography, Adv. Theor. Math. Phys. 2 (1999) 1249i [hep-th/9808014].

[25] M. Cvetič, H. Lu and C.N. Pope, Brane resolution through transgression, iNيucl. Phys.: : 